\title{
Encuentros difíciles en atención primaria: una aproximación mediante grupos focales
}

\author{
Francisco Atienza \\ UGC Adoratrices, DS Huelva-Costa
}

\author{
Luisa María Rodríguez-Fernández \\ Francisco Revuelta-Pérez \\ Universidad de Huelva
}

\author{
Carmen Losada \\ Gloria Marín \\ UGC Adoratrices, DS Huelva-Costa
}

\section{RESUMEN}

Los encuentros difíciles entre los profesionales de la salud y los pacientes producen un importante impacto emocional. Las consecuencias de esta relación disfuncional son, entre otros, aumento de la demanda asistencial y del consumo de recetas e insatisfacción laboral. El presente estudio de caso múltiple se enmarca en la dimensión relacional entre profesionales y pacientes, caracterizada por la interacción de intereses, cogniciones, emociones y conductas en el que el fin primario es la búsqueda de bien del paciente, pero que está matizado por las influencias de la triada: profesional-paciente-entorno. Los objetivos principales son describir las emociones generadas por los encuentros difíciles en atención primaria y averiguar el tipo de afrontamiento que se utiliza. La metodología empleada fue el empleo de grupos focales de discusión. Participaron en el estudio 15 médicos de familia, 12 enfermeras de familia y 6 usuarios del sistema sanitario público de Andalucía. El análisis categorial de la información recopilada en los cinco grupos focales realizados originaron tres categorías de información: magnitud percibida, emociones sentidas y causalidad atribuida. Se discuten la importancia y repercusión de estas categorías en la relación entre los profesionales de la salud y los usuarios del sistema sanitario andaluz.

Palabras clave: encuentros difíciles, relación médico-paciente, estudio cualitativo.
ABstract

Difficult encounters between health professionals and patients cause an important emotional impact. The consequences of this dysfunctional relation are, among others, an increase of the demand for health care and medical prescriptions consumption, and work dissatisfaction. The present multiple-case study is framed in the relational dimension between health professionals and patients, characterized by the interaction among interests, cognitions, emotions and behaviours, its ultimate goal being the search of patient's wellness, tinged by the influences of the professional-patientenvironment triad. The main goals are to describe the emotions generated by the difficult encounters in primary health care and to find out the copying styles that are used. The methodology implemented was the use of discussion focal groups. In this study take part 15 family practice physicians, 12 family practice nurses, and six users of the Andalusian Public Health System. The analysis of the collected information in the groups has grouped in three information categories: Perceived magnitude, experienced emotions and attributed causality. The importance and repercussion of these categories in the relation between health professionals and Andalusian public health system users are discussed.

Keywords: Difficult encounters, physicianpatient relation, qualitative research. 
La relación entre los profesionales de la salud y los pacientes busca, fundamentalmente, el intercambio de información con el fin último del beneficio de la salud de los últimos. Sin embargo, no es posible dejar de lado que esta relación conlleva un complejo entramado en el que están presentes no solo la información sino también las creencias, valores, intereses y emociones de los protagonistas de la misma. Se puede definir la relación médico-paciente (RMP) como aquella que es interpersonal, que posee connotaciones éticas, filosóficas y sociológicas de tipo profesional y que sirve de base a la gestión de salud (Rodríguez-Silva, 2006). En la RMP se produce, consecuentemente, un encuentro de sentimientos y actitudes que cada uno de los protagonistas genera durante su transcurso. Aunque el fin del encuentro clínico es la búsqueda de la salud del paciente, hay que tener presente que se produce en un contexto de intercambio de información y emociones.

El producto final de la RMP estará determinado por las diversas influencias del paciente, el profesional de la salud y el entorno en el que se realiza. En este marco puede surgir una relación distorsionada que ocasione malestar al profesional sanitario y/o al paciente, pudiéndose calificarla como encuentro difícil (ED).

La prevalencia de los encuentros difíciles en las consultas de atención primaria ha sido estudiada, entre otros, por Mas (Mas et al., 2009), mediante encuesta, encontrándose un 8,5\% (IC 95\%: 5,8-11,9) en la realizada a los médicos y un $3,1 \%$ (IC 95\%: 1,6-5,5) en la efectuada a los pacientes.

Los ED producen un importante impacto emocional en los profesionales de la salud y en los pacientes. En los primeros producen gran cantidad de sentimientos y emociones negativos, entre los que destacan la ansiedad, la rabia, la impotencia, etc. (Atienza et al., 2009), mientras que en los segundos originan insatisfacción con el servicio recibido y con la relación, así como el no cumplimiento de expectativas (Jackson \& Kroenke, 1999).

Las consecuencias de esta relación disfuncional se producen sobre los pacientes que tienen más probabilidades de sufrir peores resultados de salud (Kaplan, Greenfield, \& Ware, 1989) y de producir un mayor consumo de recetas, pruebas complementarias y demandas asistenciales (Stewart et al., 2000). El impacto de los encuentros difíciles sobre los profesionales de la salud se manifiesta en forma de insatisfacción laboral y desgaste emocional. Entre otras posibles, se provoca una disminución de interés por el trabajo, una sensación de ser manipulados y el deseo de no relacionarse más con el paciente (Smith, Lyles, \& Gardiner, 2006). Todo esto tiene relación con la aparición del síndrome de burnout caracterizado por cansancio emocional, despersonalización y desrealización (Mateen \& Dorji, 2009). Además de todo ello, la empatía en la relación médicopaciente se asocia de manera inversa con las denuncias y litigios derivados de la práctica profesional, siendo los protagonistas de los encuentros difíciles los pacientes en los que existe mayor riesgo de estas cuestiones (Moore, Adler, \& Robertson, 2000).

Aunque existen revisiones sobre la RMP (Beck, Daughtridge, \& Sloane, 2002) y también investigaciones con técnicas de grupo focal en España, sobre la calidad de la relación clí- 
nica (Girón, Beviá, Medina, \& Simón, 2002), son escasos los trabajos que analicen cualitativamente, de manera específica, los encuentros difíciles desde la perspectiva de los profesionales sanitarios, médicos y enfermeras, así como desde la de los pacientes. Un reciente estudio mediante videograbación de entrevistas clínicas y grupos focales sólo de médicos analiza la anatomía de los ED, aunque se centra más en aspectos de contenidos y duración de la consulta, así como en las estrategias de afrontamiento más que en el aspecto emocional de estos encuentros (Weingarten et al., 2010).

En el presente trabajo se ofrece una aproximación a las emociones ante los encuentros difíciles en los profesionales sanitarios (Atienza et al., 2009) y se profundiza en estos aspectos desde perspectivas diferentes, incluyendo la del paciente. Por ello, se plantea la realización de un estudio cualitativo mediante la técnica del grupo focal, para obtener información sobre la percepción de profesionales y pacientes en relación con los ED, que permita, más adelante, desarrollar herramientas que puedan evaluar de manera estandarizada los encuentros difíciles $y$, a su vez, proponer intervenciones que mejoren el abordaje profesional de estas situaciones con el menor impacto negativo posible.

Este estudio se enmarca en la relación entre profesionales y pacientes, caracterizada por la interacción de intereses, cogniciones, emociones y conductas en el que el fin primario es la búsqueda del bien del paciente, pero que está matizada por las influencias de la tríada: profesional-paciente-entorno.

Los objetivos principales son, en primer lugar, recabar información sobre las emociones que despiertan en los profesionales y pacientes los encuentros difíciles $y$, en segundo, averiguar el tipo de afrontamiento que se utiliza. Como secundarios están la obtención de información sobre la magnitud percibida de estos encuentros y las posibles estrategias de mejora de la relación clínica.

\section{Método}

\section{Participantes}

En el estudio participaron un total de 15 de médicos de familia, 12 enfermeras de familia y 6 usuarios del sistema sanitario público de Andalucía. Por lo que respecta a los profesionales sanitarios, la muestra refleja la habitual del ámbito de atención primaria en un contexto urbano. Todos tenían una amplia experiencia (mayor a los diez años de ejercicio), en su contexto laboral.

El diseño utilizado fue un estudio cualitativo (Montero \& León, 2007), mediante el empleo de grupos focales de discusión. Para ello, se formaron cinco grupos: dos con médicos de familia, dos con enfermeras de familia y uno con usuarios del un centro asistencial. Esta técnica consiste en llevar a cabo entrevistas en profundidad, en los que la interacción de los participantes da lugar a un abordaje más amplio del tema tratado, en este caso los aspectos emocionales en los encuentros difíciles. El estudio se llevó a cabo a lo largo de 2010.

\section{Instrumentos}

- Videocámara digital.

- Guión de preguntas a plantear al grupo. Se diseñaron tres plantillas adaptando las categorías a estudiar a los tres grupos de discusión: médicos, enfermeras y pacientes 
- Plantilla de recogida de datos para los observadores con las categorías estudiadas: magnitud percibida, emociones, causas y estrategias de afrontamiento.

\section{Procedimiento}

A todos los participantes se les solicitó el consentimiento informado para participar en el estudio. Los grupos tuvieron una duración aproximada de unos 90 minutos y finalizaron cuando se consideraba que sus integrantes no tenían más información que aportar. Cada grupo focal, además de los participantes, se componía de un moderador, con la función de dirigir la dinámica y actuar como dinamizador del grupo, y de dos o tres observadores que no tenían ningún papel activo, pero que recogían toda la información que se producía en la sesión, así como el lenguaje no verbal de los participantes. De todas formas, la misma se grabó en audio. Al finalizar, cada moderador, observadores y coordinadores del estudio se reunían para recopilar y ordenar la información generada.

Se decidió estudiar a todos los actores de la relación clínica -médicos, enfermeras y pacientes- para poder obtener los diferentes puntos de vista de la misma. El punto de vista del médico, como agente del diagnóstico y de la terapéutica, el de la enfermera, como facilitadora de cuidados y promotora de cambios del estilo de vida, y el del paciente, como receptor de la acción de los otros, pero también como agente activo y autónomo para la toma de decisiones en los aspectos referentes a su salud. Los participantes fueron, prácticamente, la totalidad de médicos y enfermeras del centro de salud en el que se realizó el estudio. Los pacientes se seleccionaron entre los usuarios del centro del grupo de informadores clave, cuidando que existiera representación de ambos sexos y de diversos tramos de edad. Esta selección de los pacientes se realizó así para conseguir una buena participación $y$, consiguientemente, una aportación adecuada de información. A los datos obtenidos sólo han tenido acceso cada moderador, los observadores y los coordinadores del estudio.

Tras la recogida de la información posterior a la realización del grupo focal se procedió al análisis estructurado de la información con la documentación obtenida y la aportación del material grabado. Este análisis en relación con los objetivos del estudio fue realizado por dos psicólogos y un médico de familia, expertos en la gestión de la demanda asistencial y en el análisis y categorización de emociones, y que habían participado en el estudio previo realizado mediante encuesta (Atienza et al., 2009).

En el análisis categorizado se valoró la saturación de la información que produjeron los grupos, agotándose el abanico de respuestas, así como la homogeneidad de las respuestas entre los diferentes grupos. También se valoró su correlación con la información obtenida en un estudio previo realizado mediante encuesta (Atienza et al., 2009).

\section{Resultados}

Los hallazgos obtenidos produjeron las categorías de información que siguen a continuación: magnitud percibida, emociones sentidas y causalidad atribuida. 
Tabla 1

Porcentajes de las diferentes categorías de magnitud percibida por parte de los médicos, enfermeras y pacientes

\begin{tabular}{lccc}
\hline Magnitud percibida & Médicos & Enfermeras & Pacientes \\
\hline Grande & $63,6 \%$ & $13,8 \%$ & $4,5 \%$ \\
Media & $23,6 \%$ & $36,3 \%$ & $15,6 \%$ \\
Pequeña & $13,8 \%$ & $49,9 \%$ & $79,9 \%$ \\
\hline
\end{tabular}

\section{Magnitud percibida}

Los médicos percibieron una frecuencia elevada de encuentros difíciles, aunque manifestaron que son pocos los aparecen con situaciones conflictivas. Sin embargo, para ellos la relación produce malestar. Sugirieron que al inicio de la profesión son más frecuentes, en relación al déficit de habilidades de comunicación y, también, hablaron de que cuando se comienza a trabajar con un cupo de pacientes se produce un espacio temporal con más encuentros difíciles (por ej., "es como si los pacientes nos pusieran a prueba»). Hicieron referencia a la influencia de las zonas deprimidas socioculturalmente como generadoras de encuentros difíciles pero, igualmente, advirtieron que los pacientes de mayor nivel cultural los propician por su más elevado nivel de exigencia.

Por su parte, las enfermeras señalaron que los encuentros difíciles son relativamente poco frecuentes, si bien les producen un gran impacto (por ej., «son pocos pero se notan en un simple saludo»-destacando el factor exigencia como causa generadora-, "se vuelven difíciles en el momento en el que no haces lo que ellos quieren»). Subrayaron la continuidad asistencial como elemento que disminuye los encuentros difíciles.

En cuanto a los pacientes destaca que apreciaron una frecuencia muy baja de encuentros difíciles, considerando que la relación clínica no suele generar, habitualmente, situaciones conflictivas. Existió poca percepción de que la relación podría ocasionar malestar al profesional de la salud.

En la Tabla 1 pueden verse los porcentajes para cada una de las categorías relativas a la magnitud percibida por parte de los médicos, enfermeras y pacientes.

\section{Emociones}

Los médicos refirieron que experimentan numerosas emociones en el curso de los encuentros difíciles, predominando aquellas relacionadas con la ansiedad (por ej., «me pongo muy acelerada»), angustia (por ej., «lo peor es el segundo encuentro»), miedo a perder el control y culpabilidad (por ej., "algo habré hecho mal»). También se describieron emociones negativas relacionadas con la ira, rabia e injusticia (por ej., "son los que tratamos mejor y dedicamos más tiempo») y, también, con la aversión, agresividad (por ej., «no soporto que me insulten») y, asimismo, con la tristeza, soledad, impotencia, frustración y desamparo.

Las enfermeras manifiestaron, de manera predominante, emociones negativas que describieron como ansiedad, nerviosismo, impotencia (por ej., «esto no tienen arreglo»), impaciencia, desconcierto, injusticia (por ej., « ¿por 
qué son así?»), ira (por ej., «me pongo de mala uva»), rabia por infravaloración profesional, sentimientos de culpa o autocrítica (por ej., "¿quéhe hecho mal?»), desgaste emocional (por ej., «me dan la lata»), tristeza y pena. Asimismo, señalaron como sentimientos negativos más intensos y de mayor impacto emocional el miedo y la angustia. Por otra parte, también afloraron emociones positivas de comprensión (por ej., «soy el profesional y tengo que entender al paciente») y de superación (por ej., «tengo que preparar mejor la consulta»).

Por su parte, los pacientes describieron, mayoritariamente, sentimientos de agresividad (por ej., «dan ganas de cogerle por el cuello» o «quisiera darle un tortazo»), pero, también, de impotencia, rabia, malestar y ganas de llorar.

Similarmente, en la Tabla 2, aparecen los porcentajes correspondientes a este aspecto de las emociones.

\section{Causas}

Los médicos pensaron que la causa predominante que da origen a los encuentros difíciles es la personalidad de los pacientes -especialmente aquellas que etiquetaron como exigentes y agresivas-, los problemas de salud mental, la situación personal (por ej., «ellos pueden tener un mal día») y las expectativas poco fundamentadas, (por ej., "ellos vienen a que les demos una pastilla para que encuentren trabajo»). También hicieron referencia al exceso de información poco cribada obtenida a través de Internet, así como al fenómeno inmigratorio con las expectativas excesivas que tienen los inmigrantes en relación a sistema sanitario de acogida, lo que los hace hiperdemandantes y poco respetuosos con las normas de funcionamiento del sistema. Asimismo, comentaron el factor del profesional en relación con los excesos de cargas de trabajo, especialmente algunos días y en determinadas horas específicas (al principio y sobre todo al final de la jornada), el estilo de afrontamiento y la experiencia de los profesionales y factores de la organización en relación con los profesionales como el poco tiempo por paciente o las dificultades en la continuidad asistencial, lo que da lugar a una relación clínica de baja calidad.

Las enfermeras hicieron referencia a expectativas equivocadas o exageradas y las características personales de los pacientes (por ej., «los pacientes de metadona son impacientes y cronifican una relación difícil», «piensan que tenemos que darle una solución a su problema al instante»). Las características de los pacientes más referidas fueron: personas que no escuchan -solo vienen a hablar ellos-, acompañantes que consultan sin cita, pacientes de difícil trato y no sólo en la consulta -esos son así-, mala educación, hiperfrecuentadores, violentos, agresivos, con demandas injustificadas y amenazantes (por ej., "como no me hagas la prueba del azúcar y tenga diabetes, te vas a enterar»). En relación a circunstancias de los profesionales manifestaron la infravaloración de la profesión enfermera, la falta de habilidades de comunicación y las personalidades rígidas y poco flexibles. También hablaron de circunstancias del entorno que pueden propiciar los ED, tales como errores en la información, dificultades de comunicación entre los profesionales (especialmente entre médicos y enfermeras), falta de tiempo y variabilidad de la práctica clínica 
Tabla 2

Porcentajes de las diferentes categorías de emociones por parte de los médicos, enfermeras y pacientes

\begin{tabular}{lrcr}
\hline Emociones & Médicos & Enfermeras & Pacientes \\
\hline Ansiedad & $52,4 \%$ & $54,3 \%$ & $10,6 \%$ \\
Angustia & $46,9 \%$ & $38,3 \%$ & $10,6 \%$ \\
Miedo a perder el control & $9,9 \%$ & $36,3 \%$ & $0,0 \%$ \\
Culpa & $23,6 \%$ & $18,7 \%$ & $0,0 \%$ \\
Ira & $38,9 \%$ & $24,3 \%$ & $36,7 \%$ \\
Rabia & $38,9 \%$ & $44,3 \%$ & $59,6 \%$ \\
Injusticia & $57,1 \%$ & $65,3 \%$ & $61,9 \%$ \\
Aversión & $40,2 \%$ & $47,6 \%$ & $8,6 \%$ \\
Agresividad & $38,9 \%$ & $36,3 \%$ & $72,6 \%$ \\
Tristeza & $18,7 \%$ & $24,7 \%$ & $22,3 \%$ \\
Soledad & $12,9 \%$ & $46,9 \%$ & $0,0 \%$ \\
Impotencia & $51,3 \%$ & $48,9 \%$ & $68,7 \%$ \\
Frustración & $30,9 \%$ & $47,6 \%$ & $58,9 \%$ \\
Desamparo & $16,6 \%$ & $12,2 \%$ & $0,0 \%$ \\
Desgaste emocional & $18,7 \%$ & $16,4 \%$ & $0,0 \%$ \\
Comprensión & $7,6 \%$ & $12,2 \%$ & $0,0 \%$ \\
Superación & $6,3 \%$ & $16,4 \%$ & $0,0 \%$ \\
\hline
\end{tabular}

Los pacientes pensaron que las causas de los ED son, fundamentalmente, las características del profesional sanitario que los atiende tales como la falta de empatía, la escasa atención (por ej., «no se preocupan de nuestros problemas»), la resistencia a satisfacer sus demandas (por ej. «no nos mandan al especialista cuando se lo pedimos»), la falta de habilidades de comunicación (por ej., «a veces no son capaces de identificar nuestra demanda») y, también, la falta de confidencialidad y algunas características personales como la poca sensibilidad hacia los problemas de los pacientes, el déficit de educación social, el trato desagradable o la falta de ética profesional. También hicieron mención de factores de contexto como la presencia, a veces solos, de médicos residentes, los fallos informáticos o la falta de destreza de los profesionales, que ralentiza el tiempo de atención y la falta de tiempo de atención. No olvidaron mencionar algunos factores de los pacientes como causantes de ED como la falta de cultura, de educación y algunas variables de personalidad (conflictivos, agresivos).

En la Tabla 3 se ofrecen los porcentajes de las distintas categorías referidas a las causas.

\section{Afrontamiento}

Los médicos refirieron tipos de afrontamiento como la reducción del tono emocional, hablando bajo, con tono neutro, extremando la cortesía (por ej., «siéntese, por favor», «cierre la puerta, por favor») e intentando el control de la situación. Otros afrontamientos intentan la 
Tabla 3

Porcentajes de las diferentes categorías de causas por parte de los médicos, enfermeras y pacientes

\begin{tabular}{lccc}
\hline Causas & Médicos & Enfermeras & Pacientes \\
\hline Personalidad del paciente & $86,9 \%$ & $66,3 \%$ & $0,0 \%$ \\
Problemas de salud mental & $46,9 \%$ & $22,4 \%$ & $0,0 \%$ \\
Situación personal & $33,6 \%$ & $40,3 \%$ & $18,9 \%$ \\
Expectativas no cubiertas & $43,9 \%$ & $78,9 \%$ & $66,8 \%$ \\
Carga de trabajo & $28,7 \%$ & $38,9 \%$ & $0,0 \%$ \\
Dificultades de comunicación & $18,9 \%$ & $24,7 \%$ & $0,0 \%$ \\
Variabilidad de la práctica clínica & $12,9 \%$ & $24,7 \%$ & $0,0 \%$ \\
Falta de empatía & $12,9 \%$ & $0,0 \%$ & $18,9 \%$ \\
Escasa atención & $0,0 \%$ & $0,0 \%$ & $18,9 \%$ \\
Resistencia a satisfacer demandas & $0,0 \%$ & $0,0 \%$ & $68,7 \%$ \\
Personalidad del profesional & $7,6 \%$ & $0,0 \%$ & $89,9 \%$ \\
Entorno de la atención & $38,9 \%$ & $33,6 \%$ & $22,6 \%$ \\
\hline
\end{tabular}

racionalización de la situación y la búsqueda del error cometido por el profesional o de las diferencias entre las expectativas del paciente y la realidad asistencial. Por último, también existen afrontamientos negativos como la evitación, huida (por ej., "o te vas tú o me voy yo»), el evitar la comunicación verbal y no verbal (por ej., "me pongo a escribir en el ordenador para no mantenerle la mirada»), la proposición de cambio de médico o el bloqueo emocional. Por otra parte, se comentó la necesidad de tomarse un respiro emocional y físico tras un ED conflictivo, para poder continuar la consulta y el análisis a posteriori de los incidentes para metabolizarlos.

Los enfermeras plantearon estrategias de afrontamiento de tipo de autocontrol y pensamientos positivos (por ej., "yo controlo», «necesito controlar la ansiedad») y, también, de distanciamiento y reducción del tono emocional (disco rayado, mantener la autoridad, mantener la distancia, ser asertivo, bajar la voz, actuar de pantalla). Asimismo, nombraron estrategias activas como escucha activa, negociación, extremar la amabilidad, evitar el uso de imperativos, hacer que los pacientes verbalicen sus problemas o empatizar con el paciente. En estos profesionales también aparecieron afrontamientos negativos como la huida, (por ej., «me levanto y me voy», «me quito de en medio»). A posteriori estos profesionales también mencionaron la realización de análisis de los ED, especialmente los conflictivos, para prevenir la aparición de los mismos.

En los pacientes el afrontamiento realizado por los pacientes consistió en la pasividad (por ej., «bajamos la cabeza y nos vamos para casa»), el cambio de médico y, en casos extremos, la reclamación o la denuncia.

En la Tabla 4 se muestran los porcentajes de las diferentes categorías obtenidas en relación con el afrontamiento. 
Tabla 4

Porcentajes de las diferentes categorías de afrontamiento por parte de los médicos, enfermeras y pacientes

\begin{tabular}{lrcc}
\hline Afrontamiento & Médicos & Enfermeras & Pacientes \\
\hline Reducción del tono emocional & $86,9 \%$ & $65,3 \%$ & $0,0 \%$ \\
Racionalización y búsqueda del error & $46,9 \%$ & $24,7 \%$ & $0,0 \%$ \\
Evitación y/o huida & $6,3 \%$ & $16,7 \%$ & $0,0 \%$ \\
Respiro emocional & $18,9 \%$ & $24,7 \%$ & $0,0 \%$ \\
Autocontrol & $46,9 \%$ & $38,9 \%$ & $0,0 \%$ \\
Negociación & $12,9 \%$ & $22,4 \%$ & $0,0 \%$ \\
Empatía & $12,9 \%$ & $12,2 \%$ & $0,0 \%$ \\
Pasividad & $0,0 \%$ & $0,0 \%$ & $89,9 \%$ \\
Reclamación & $0,0 \%$ & $0,0 \%$ & $36,7 \%$ \\
\hline
\end{tabular}

\section{Discusión}

En primer lugar, hay que indicar que la elaboración de este estudio en el ámbito urbano puede condicionar la pérdida de información respecto al impacto de la realización de guardias y atención urgente sobre la aparición de encuentros difíciles. Asimismo, el hecho de que la mayoría de los profesionales participantes tuvieran una experiencia laboral superior a los quince años puede originar una cierta distorsión de los resultados. Sin embargo, los hallazgos obtenidos presentan coherencia con la metodología empleada, siendo destacable la homogeneidad de los resultados en los grupos de profesionales y su convergencia con una encuesta previa (Atienza et al., 2009).

La prevalencia de ED ha sido analizada en varios estudios (Mas et al., 2009; MartínezCañavate et al., 2004; Prados et al., 1990), encontrándose unos valores que oscilan entre el $5,6 \%$ y el $9,4 \%$. La magnitud percibida por los profesionales no es, en principio, muy elevada en el presente estudio, aunque sí se hace referencia al gran desgaste que producen; sin embargo, al profundizar en el aspecto de ma- lestar como definición del ED, esta magnitud se incrementa notablemente. Este hecho debe hacer reflexionar sobre la necesidad, ya expresada por otros autores (Ballesta, 2010), de unificar los criterios y herramientas de medida de los ED, lo que permitiría la estandarización y la comparación entre los diferentes estudios. Asimismo, en el trabajo aquí expuesto aparece una discrepancia entre la magnitud de los ED percibida por los profesionales y la relativa a los pacientes (menor que la de aquellos) y que podría relacionarse con la escasa identificación, por parte de los pacientes, del profesional sanitario como persona en contraste con la que sí tienen como técnico y prestador de los servicios demandados por ellos, lo que explicaría la sensación de sorpresa que experimentan ante un ED y sus reacciones de agresividad.

Este estudio muestra cómo las emociones principalmente sentidas por los profesionales se relacionan con la ansiedad, la rabia o impotencia y el sentimiento de injusticia. Estos resultados son concordantes con los de otras investigaciones, como la de Ballesta (2010) que identificó como principales emociones 
sentidas por 45 médicos de familia que habían tenido un ED, ansiedad e impotencia en primer lugar, desconfianza-ira en prevalencia intermedia y la inseguridad, depresión y emociones positivas de superación en un rango más bajo.

En nuestro estudio también aparece el miedo y la angustia y, también, con más frecuencia sentimientos positivos de autocontrol y profesionalidad. Por otro lado, es de resaltar en ambos profesionales sanitarios, si bien especialmente en los médicos, el alto contenido emocional expresado a través del lenguaje no verbal que se produjo al hablar de las emociones.

Es destacable el hecho de que las emociones más generalizadas en los pacientes son la rabia, la impotencia y la agresividad hacia el profesional, que podría estar en la base de las conductas de agresión a profesionales que vivimos, aunque de manera esporádica, en el ejercicio profesional.

Como ya se ha puesto de manifiesto en otros estudios (Martínez-Cañavate et al., 2004; Mas et al., 2009) la atribución causal a las características del otro elemento implicado en la relación, se produce en nuestro estudio. Los profesionales sanitarios piensan que son las características del paciente las que definen la aparición de ED, mientras que los pacientes aseveran que son las del profesional las que determinan estos ED. Es de señalar la aparición de elementos organizativos, en sentido amplio, como la información sobre las características del servicio sanitario, las expectativas poco fundadas de los pacientes o la falta de tiempo o de capacidad técnica apuntadas por los pacientes. Parece claro que la triada paciente-profesional-entorno, interactúa en la aparición de ED y esto se percibe por todos los actores aunque tiñendo esta visión por su perspectiva propia.

Las respuestas obtenidas en relación con los afrontamientos de los ED por los profesionales, apunta a una deficiencia en las habilidades de manejo de los mismos, destacando los afrontamientos de intento de control emocional pero, también, de evitación o huida o, incluso, de proyección de la agresividad hacia el paciente. El estudio de Ballesta (2010) apunta al neuroticismo, medido por el cuestionario EPQ de Eysenck (1991), como elemento predictor de emociones negativas ante los ED en los profesionales.

Parece necesario iniciar estudios que investiguen la efectividad de intervenciones basadas en los aspectos cognitivo-comportamentales, tanto tradicionales como las de llamadas de tercera generación -como son, por ejemplo, la terapia racional emotiva conductual de Ellis (Ellis \& Grieger, 1990), la terapia de aceptación y compromiso (ACT) (Luciano, Valdivia, Gutiérrez, \& Páez, 2006) o la mindfullness (Siegel, 2009)- en la disminución del desgaste producido por los ED. La adquisición de mayores habilidades de comunicación y de técnicas de entrevista clínica podrían contribuir a minimizar el impacto negativo de los ED.

Este estudio intenta dar respuesta a los objetivos planteados sobre el conocimiento de las emociones generadas por los ED y los estilos de afrontamiento de los mismos. Se espera que este conocimiento pueda contribuir al desarrollo de futuras investigaciones sobre 
intervenciones efectivas aplicadas a los profesionales de la salud, especialmente en la línea de la terapia cognitivo-comportamental que mejoren la vivencia y aceptación de estas situaciones.

\section{Referencias}

Atienza Martín, F. J., Revuelta Pérez, F., Santana Gallego, M., Bermúdez Jiménez, R., Lozano Cano, M., \& Romero Ruiz, B. (2009, noviembre). Encuentros difíciles en Atención Primaria: sentimientos de los profesionales y atribuciones causales. Póster presentado en el XXIX Congreso de la Sociedad Española de Medicina Familiar y Comunitaria, Barcelona.

Ballesta Rodríguez, M. I. (2010). Respuesta emocional de los médicos de familia en los encuentros médico-paciente difíciles. Tesis doctoral no publicada. Universidad de Granada, Granada.

Beck, R. S., Daughtridge, R., \& Sloane, P. D. (2002). Physician-patient communication in the primary care office: A systematic review. The Journal of the American Board of Family Practice, 15, 25-38.

Ellis, A., \& Grieger, R. (1990). Manual de terapia racional emotiva (M. a J. Catalán Frías \& J. D. González Díez, Trads.). Bilbao: Editorial Desclée de Brouwer. (Trabajo original publicado en 1977).

Eysenck, H. J. (1991). Eysenck Personality Scales. Londres: Hodder \& Stoughton.

Girón, M., Beviá, B., Medina, E., \& Simón, M. (2002). Calidad de la relación médico paciente y resultados de los encuentros clínicos en atención primaria de Alicante: un estudio con grupos focales. Revista Española de Salud Pública, 76, 561-575.

Jackson, J. L., \& Kroenke, K. (1999). Difficult patient encounters in the ambulatory clinic: clinical predictors and outcomes. Archives of Internal Medicine, 159, 1069-1075.
Kaplan, S. H., Greenfield, S., \& Ware, J. E. (1989). Assessing the effects of physician-patient interactions on the outcomes of chronic disease. Medical Care, 27, 110-127.

Luciano, M. C., Valdivia, S., Gutiérrez, O., \& Páez, M. (2006). Avances desde la Terapia de Aceptación y Compromiso (ACT). Revista de Psicología y Psicopedagogía, 5, 173-201.

Martínez-Cañavate, T., Bellón, J. A., Delgado, A., Luna, J., López, L.; \& Lardelli, P. (2004). Modelo multinivel explicativo de la utilización de las consultas de atención primaria a partir de los factores del profesional y de la organización. Atención Primaria, 34, 119-155.

Mas, G. X., Navarro, G. M., Vázquez, G. M., Delso, G. C., Mahfouz, C. T. , \& Alameda, O. J. (2009). Encuentros difíciles en consultas de atención primaria: una perspectiva de pacientes y médicos. Atención Primaria, 41, 9-15.

Mateen, F. J., \& Dorji, C. (2009). Health-care worker burnout and the mental health imperative. Lancet, 374, 595-597.

Montero, I., \& León, O. G. (2007). A guide for naming research studies in Psychology. International Journal of Clinical and Health Psychology, 7, 847-862.

Moore, P. J., Adler, N. E., \& Robertson, P. A. (2000). Medical malpractice: the effect of doctor-patient relations on medical patient perceptions and malpractice intentions. The Western Journal of Medicine, 173, 244-250.

Prados, J. A., Pérez, A., Muñoz, F., Gómez, F., Gálvez, L., \& Guerra, C. (1990, diciembre). Frecuencia y características socioculturales del paciente problema en las consultas a demanda.Póster presentado en la European Regional Conference on Family Medicine, WONCA I. Barcelona.

Rodríguez-Silva, H. (2006). La relación médicopaciente. Revista Cubana de Salud Pública, 32(4). Recuperado de http://scielo.sld.cu/ scielo.php?script $=$ sci_arttext $\&$ pid $=$ S086434662006000400007\&lng=es\&nrm=iso\&tlng=es 
Siegel, R. (2009). The mindfulness solution: Everyday practices for everyday problems. New York: Guilford Press.

Smith, R. C., Lyles, J. S., Gardiner, J. C., Sirbu, C., Hodges, A., Collins, C.,...Goddeeris, J. (2006). Primary care clinicians treat patients with medically unexplained symptoms: a randomized controlled trial. Journal of General Internal Medicine, 21, 671-677. Doi:10.1111/j.1525-1497.2006.00460.x

Stewart, M., Brown, J. B., Donner, A., McWhinney, I. R., Oates, J., Weston, W.W., \& Jordan, J. (2000). The impact of patient-centered care on outcomes. Journal of Family Practice, 49, 796-804.

Weingarten, M. A., Guttman, N., Abramovitch, H., Margalit, R. S., Roter, D., Ziv, A., \& Borkan, Y. J. (2010). An anatomy of conflicts in primary care encounters: A multi-method study. Family Practice, 27, 93-100. 